\title{
A Machine Learning Approach to Tongue Motion Analysis in 2D Ultrasound Image Sequences
}

\author{
Lisa Tang $^{1}$, Ghassan Hamarneh ${ }^{1}$, and Tim Bressmann ${ }^{2}$ \\ 1 Medical Image Analysis Lab., School of Computing Science, Simon Fraser University \\ ${ }^{2}$ Department of Speech-Language Pathology, Faculty of Medicine, University of Toronto
}

\begin{abstract}
Analysis of tongue motions as captured in dynamic ultrasound (US) images has been an important tool in speech research. Previous studies generally required semi-automatic tongue segmentations to perform data analysis. In this paper, we adopt a machine learning approach that does not require tongue segmentation. Specifically, we employ advanced normalization procedures to temporally register the US sequences using their corresponding audio files. To explicitly encode motion, we then register the image frames spatio-temporally to compute a set of deformation fields from which we construct the velocity-based and spatiotemporal gestural descriptors, where the latter explicitly encode tongue dynamics during speech. Next, making use of the recently proposed Histogram Intersection Kernel, we perform support vector machine classification to evaluate the extracted descriptors with a set of clinical measures. We applied our method to speech abnormality and tongue gestures prediction. Overall, differentiating tongue motion, as produced by patients with or without speech impediments on a dataset of 24 US sequences, was achieved with classification accuracy of $94 \%$. When applied to another dataset of 90 US sequences for two other classification tasks, accuracies were $86 \%$ and $84 \%$.
\end{abstract}

\section{Introduction}

In recent decades, ultrasound imaging research has made great strides capturing the intricate and highly coordinated nature of articulatory movements for speech. However, the tongue makes complex motion and complicated postural adjustments during speech production, making perceptual assessments of speech problems from US images extremely challenging.

Accordingly, the development of reliable and robust quantitative indicators for what constitutes normal tongue movement or what characterizes articulation errors and/or speech disorders is highly desirable. These indicators would especially be useful to inform treatment strategies for speech-language pathologists (SLP) [1] as these indicators would provide more explicit information of the tongue motion (e.g. which parts of the tongue have moved incorrectly and how incorrectly).

As a first step in developing these indicators, this paper employs a learning approach to analyze mid-sagittal tongue motion as captured in US image sequences and, in particular, seeks to develop a descriptor that can capture spatio-temporal tongue gestures, i.e. the dynamics of the tongue contour over time. For this task, our method employs 
non-rigid registration where motion fields describing pixel displacements between consecutive frames are used to construct descriptors that encode tongue trajectories as produced during speech. We then train a support vector machine (SVM) classifier using paired data to predict a set of known measures using our proposed descriptors.

There exist several related works that examined image-captured motions for clinical problems, albeit a majority was developed specifically for cardiac motion analyses. In heart motion abnormality detection, for example, researchers have employed shape-based, tensor-based, and motion-based descriptors to study the dynamics of myocardium deformation [2]. However, direct application of these methods to tongue motion analysis is challenging due to the lack of image features for locating tongue surfaces. Due to US wave reflectance, for instance, parts of the tongue may appear missing in a frame. As 2D US sequences capture only the mid-sagittal portion of a tongue, it is often the case that the entire tongue appears as missing. Tongue motion in daily speech also does not exhibit regularity, but rather consist of intricate tongue "gymnastics". All of these issues render reliable tongue contour detection and tracking extremely difficult.

For analysis of tongue motions from US images, most of the previous studies $[3,4$, $5,6]$ only examined tongue motions using velocity measurements that relied on manual delineation of tongue contours, which is both labourious and difficult [7]. There exist a few studies that employed machine learning algorithms, e.g. [8,9], which nevertheless did not examine spatio-temporal gestures like we do and had very different applications than ours.

In contrast to the above references, our approach not only analyzes horizontal and vertical displacements, but also examines motion patterns. To develop robust descriptors, we make use of the accompanying audio files to perform temporal alignment of the US sequences. We then employ a well-validated registration algorithm [10] to reliably obtain a set of spatial correspondences that explicitly represent motion and subsequently extract a set of descriptors to capture dynamics of the tongue from the set of correspondences. In evaluating the effectiveness of the descriptors, we performed three clinically driven classification tasks. As we will show in our experimental results, our approach is capable of analyzing image sequences that contain a much wider variety of utterances than those encountered in previous studies $[11,12,4,5,6]$.

\section{Methods}

Two sets of data were collected in this study, each of size $N$. In each study $n$, both audio recordings $A_{n}$ and US imaging $U_{n}$ were acquired while a subject recited a passage or articulated a sequence of utterances. Details on how the data is used are given in Sec. 3.

Our approach begins with the normalization of the data, which is an important step to ensure that the descriptors we extract are comparable across subjects. In our problem, the normalization step involves the selection of a set of keyframes in which the same set of utterances was articulated by different subjects. For this task, we perform temporal alignment on all $N$ image sequences $U$ by performing Dynamic Time Warp (DTW) on all audio recordings. With the keyframes extracted for each sequence, we then perform spatio-temporal registration to obtain a set of deformation fields from which we extract our descriptors. From the obtained deformation fields that describe tongue motions with 
respect to a reference frame, we next extract a set of measurements from each field and encode these measurements with a histogram-based representation to ensure robustness against outlier displacement vectors. Lastly, we train a specialized SVM classifier to learn possible correlations between the features and a set of known clinical measures.

\subsection{Audio-based temporal alignment via dynamic time warp}

As explained earlier, normalization of the data is crucial to ensure that extracted features are meaningful and comparable across subjects. In our US data, because reading speeds varied across subjects, the frame number that corresponds to the same sound can be different across subjects. This called for temporal normalization. Only after we have resolved the temporal misalignments that exist between US sequences can we attribute the differences in our overall training data to differences in tongue gestures.

Correcting temporal misalignments based on the US images is extremely difficult as changes in appearance features between frames are very subtle. Conversely, distinctive temporal landmarks can be easily identified in the audio signals that accompany the US data. We thus used the audio signals to perform temporal registration of the US frames. Firstly, one of the sound files in each dataset is chosen as the audio template. Next, we employ the Dynamic Time Warping (DTW) algorithm of [13] (based on dynamicprogramming) to temporally align each extracted sound originating from the template to those matched in the remaining sequences. To align two audio signals, DTW operates in the space of the time frames constructed with respect to the two sequences and seeks a path through this space that maximizes the local match between the aligned time frames, subject to some ordering constraints. The sum of these local similarity costs is then used to estimate how well two signals match. Temporal registration of each sequence with respect to the template consequently yields a set of time points that indicate when the same sounds were made across all subjects. Based on the frame rate of the captured US sequences, we calculate, for each sequence $n$, a set of $k$ frame indices that essentially provide the temporal correspondences in $U$. However, converting from time to frame number $I_{n}$ may result in a frame index that falls in the space between two US frames. In this case, linear interpolation on such pair of frames was done. Finally, extracting a subset from $U_{n}$ based on $I_{n}$ yields $U_{n}^{*}$ that are temporally aligned across subjects.

\subsection{Explicit characterization of dynamic gestures via 2D+time registration}

In [9], image features were used as descriptors to differentiate static tongue gestures. However, as our goal is to differentiate between different dynamic tongue gestures (spatio-temporal vs. just spatial gestures [9]), we need to capture the motion/gestural changes that occur across time. Accordingly, we performed spatio-temporal registration on each of $U_{n}^{*}$ to explicitly find a set of inter-frame per-pixel correspondences, from which we extract information to capture tongue motions and gestural changes.

For this task, we employ the latest state-of-art registration method of Metz et al. [10] that enforces both spatial and temporal smoothness on sought deformation fields; the latter in particular would help decrease the sensitivity of registration to sporadic disappearances of image features of the tongue due to US wave reflectances, etc. (Fig. 1a). 

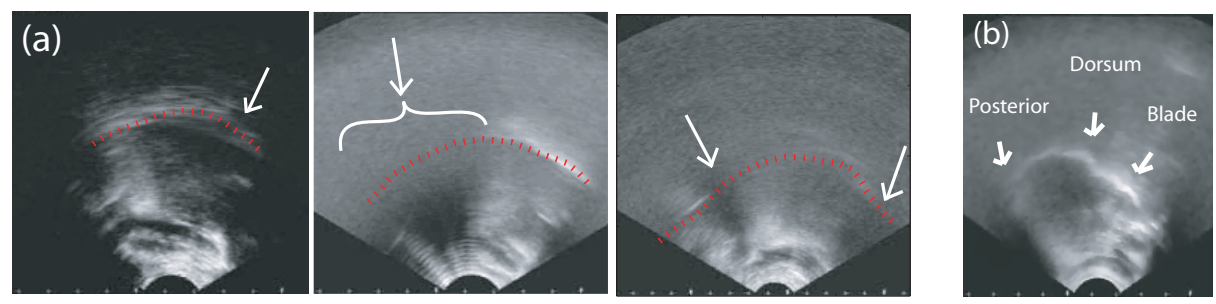

Fig. 1. (a) US frames showing missing parts of the tongue. (b) Diffferent tongue regions.

This algorithm was designed to solve the parameters of a Lagrangian 2D+time transformation model that would minimize the intensity variance at corresponding spatial locations across time.

\subsection{Feature extraction}

Spatio-temporal registration on all $N$ US subsequences of length $k$ results in a set of displacement vector fields $\left\{\mathbf{D}_{(s, 1)}, \mathbf{D}_{(s, 2)}, \ldots, \mathbf{D}_{(s, i)}, \ldots, \mathbf{D}_{(s, k-1)}\right\} \forall s=1: n$, each of which describes the coordinate mapping from frame $i$ to frame $i+1$. From these, we calculated a set of descriptors of two types which we detail below.

Regional velocity-based descriptors. Following [3], which concluded that velocity is an indicator for normal and abnormal tongue motion, we have built our descriptors based on velocities.

In [11], a hard threshold was applied on the motion vectors of each field such that only displacement vectors with magnitude above the mean were considered. We argue that this approach can easily discard potentially relevant information and that analysis on the remaining vectors would subsequently depend on the threshold chosen. As a better alternative, we employ histograms to encode all available information in the deformation fields. Specifically, we compute the distributions of the $x$ - and $y$-components of each velocity vector to construct our histogram-based descriptors which we denote as $V_{x}$ and $V_{y}$.

Furthermore, it is of clinical interest to examine whether differences in features at different parts of the tongue exist as this may give implications to impediments of specific muscles. Therefore, we have regionalized the encoding of features by extracting the above features in terms of the left, middle, and right sides of the image domain, which corresponds to regions of the tongue blade, dorsum and posterior (Fig. 2b). For brevity, we append each feature type with a suffix 'B', 'D', or 'P' (e.g. $\left.V_{x}-D\right)$ to denote the blade, dorsum, and posterior, respectively. Each feature type was encoded with $h=$ 20 histogram bins, where $h$ was chosen based on initial experiments.

Spatio-temporal gestural descriptors. We have developed these descriptors to explicitly encode changes in motion over time. Rather than treating each displacement vector in a field as a feature component, which would involve a feature vector of size equal to the product of the image dimensions, $d$, we perform dimensionality reduction to encode 
the most relevant information in each field. To compactly represent the obtained displacement vector field of each frame, we employ principal component analysis (PCA) to project the displacement fields onto a feature space from which we obtain a finite set of orthogonal principal components that would constitute, up to a certain accuracy, a subspace for the representations of the most likely configurations of $x$ and $y$ displacements. Subsequently, the $x$ and $y$ components in each field are represented compactly in terms of their projections onto the computed set of principal components. Note that while this dimensionality reduction step is similar to the EigenTongue feature space of [8], our approach operates on the displacement vector fields, rather than the original static US image frames of the tongue. For brevity, we denote the principal coefficients describing the projections of frame $i$ as $\mathbf{P}_{x}^{i}, \mathbf{P}_{y}^{i}$ for $x$ and $y$ dimension, respectively. With the principal coefficients representing each field computed, we then concatenate these coefficients of all frames in each US subsequence to form our gestural descriptor, i.e. $\left[\mathbf{P}_{x}^{1} \mathbf{P}_{y}^{1} \ldots \mathbf{P}_{x}^{k} \mathbf{P}_{y}^{k}\right]$. Note that we use the first $p<<d$ principal component coefficients corresponding to the first $p$ principal components that account for $98 \%$ of the variance in all vector fields.

\subsection{Training the SVM classifier}

Our next step is to train a SVM classifier to build a model that correlates the extracted features to a set of given measures (to be given in Sec. 3). Prior to training and classification, each component of all feature vectors is normalized to [0,1].

In SVM, the classifier simultaneously minimizes the classification error and maximizes the margin between classes [14]. In measuring distances between our descriptors, we employ the Histogram Intersection Kernel (HIK) that was recently proposed by $\mathrm{Wu}$ [14]. Aside from being computationally efficient, it was empirically shown to be insensitive to the $C$ parameter in SVM and is capable of achieving a higher accuracy in SVM classification than linear or radial basis function kernels in various application domains [14]. Under this kernel, the distance between two vectors $a$ and $b \in \mathbb{R}^{r}$ is defined as $\mathcal{K}(a, b)=\sum_{j=1}^{r} \min \left(a_{j}, b_{j}\right)$.

In training the SVM classifier, we employ the deterministic Intersection Coordinate Descent (ICD) algorithm that was also proposed by $\mathrm{Wu}$ [14], which solves the classification problem without re-encoding the input data and explicitly finds the feature space decision boundary.

\section{Experiments}

Accuracy of tongue tracking directly affects the sensitivity of the extracted features for our learning task. It is therefore important to assess the accuracy of the spatio-temporal registration results. To access accuracy, 12 randomly selected registration results were validated. From each sequence, two sets of manually created tongue segmentations were acquired and subsequently treated as bronze-standard. Segmentation of the first keyframe was then selected as the template and was 'propagated' to the subsequent frames using the obtained deformation fields. We then computed the mean distances between the propagated template with the bronze-standard segmentations. Fig. 2a shows 
(a) Distribution of Mean Distances

(b) Registration error per frame
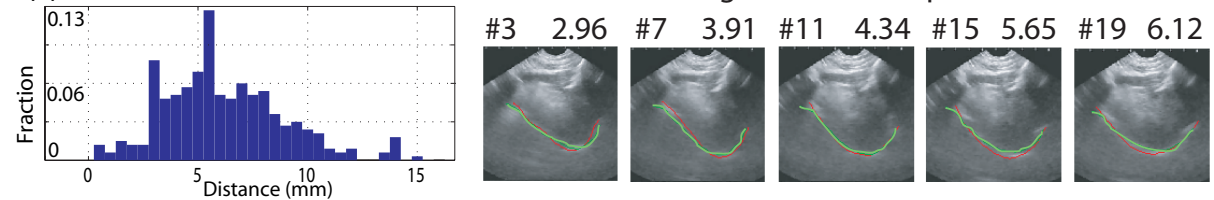

Fig. 2. Evaluation of registration results. Distribution of (a) mean distances between the obtained and expert-delineated contours in all segmented frames. (b) One registration result showing expert-delineated tongue contours (in green) and those obtained via registration (in red). Error (in $\mathrm{mm}$ ) noted on top of each image, after each frame number.

the distribution of the mean Euclidean distances between the propagated and bronzestandard contours in all segmented frames. Overall, the mean Euclidean distance was $5.82 \mathrm{~mm}$. This is acceptable as it compares well with the inter-operator segmentation variability of $5.3 \mathrm{~mm}$, which we measured based on segmentation results from two operators. Fig. $2 \mathrm{~b}$ shows an example of the propagated and bronze-standard segmentations.

Having validated the registration results, we then extracted the proposed features from the obtained fields. Next, we performed two experiments to evaluate our approach for tongue motion analysis. The first experiment examines how well our velocity-based descriptors can predict normal vs. abnormal speech. The second experiment questions whether different tongue motions can be effectively encoded with our gestural descriptors to such an extent that they can be used to predict different tongue gestures.

Differentiating normal from abnormal. The first experiment examined how our velocitybased descriptors can differentiate tongue motions produced from patients with and without speech impediments. For this experiment, we employed the data from [3], which consists of 12 studies from patients with normal speech and 12 studies from the same patients with abnormal speech (before and after undergoing lateral partial glossectomies). Each study consists of an audio recording and an US image sequence of 200-420 frames that captures the patient's tongue motion while reading a passage.

For motion analysis, we extracted $k=120$ keyframes from each of the 24 US image sequences, thus giving rise to a set of $k \times 24$ frame samples of tongue motion, each associated with a label of 'normal' or 'abnormal'. We then performed temporal registration on the extracted keyframes for each patient study to obtain a set of displacement vector fields from which we extracted the features described in Sec. 2.3. Next, we trained a SVM-classifier to build a model that would predict the type of speech impediments from the set of extracted features.

We now report the 'with vs. without speech impediments' mean classification accuracies ${ }^{1}$ of individual features as obtained from a 10 -fold cross validation experiment.

\footnotetext{
${ }^{1}$ For each classification task, we ran 12 repeated trials, each with a different value of $C$ (parameter in SVM) within $\left[2^{-8}, 2^{8}\right]$ and computed accuracy for each trial as the fraction of correctly classified samples over all samples. We then report accuracy as the mean over all 12 trials.
} 
For the velocity-based features, these were $86,90,81,89,80$, and $81 \%$ for $V_{x}-B, V_{y}-B$, $V_{x}-D, V_{y}-D, V_{x}-P$, and $V_{y}-P$ respectively. This result is in agreement with the results obtained in [3], which showed that velocity was an indicator for abnormal speech, and is an encouraging one as it reflects that our approach gave results identical to a method that relied on manual segmentation. Furthermore, the mean classification accuracy of $\mathrm{Vy}-\mathrm{B}$ was $9 \%$ greater than the accuracy of Vy-P, suggesting that the $y$-velocity exhibited in the blade region differentiated between normal and abnormal tongue motions much more effectively than those exhibited in the posterior region. Using all velocity-based features simultaneously, we achieved mean classification accuracy of $94 \%$.

Differentiating utterance types using the gestural descriptors. Our next task examined whether the gestural descriptors can describe different motion patterns as produced by articulations of different utterances. We have done so by quantifying how well they can be used to predict 3 tongue gestures produced by articulations of 3 distinct utterances.

The data for this experiment originated from [15] and involved 9 subjects articulating three vowel-consonant-vowel (VCV) sequences. These are /aka/, /ishi/, /ushu/. Generally, articulation of each VCV sequence by all subjects involved different spatiotemporal tongue gestures, with /aka/ having the most pronounced lingual excursion, while /ishi/ and /ushu/ have relatively much less movements. In fact, from inspection of the US images, the tongue gestures of the latter two appeared similar, making the gesture-prediction task challenging.

Each subject recited the same VCV sequence 10 times consecutively before proceeding to the next sequence, yielding a set of $9 \times 10$ motion samples. After performing the normalization step outlined in Sec. 2, each motion sample consisted of $k=30$ frames. We then extracted the gestural descriptors and applied them to 4 gestureprediction tasks: a 3-class classification task and three binary classification tasks (/aka/ vs. /ishi/, /ishi/ vs. /ushu/, and /aka/ vs. /ushu/). Mean classification accuracies for the 4 respective tasks were $74,86,86$, and $84 \%$. While classification accuracy for the 3class problem was suboptimal, all binary classifications gave comparable performances, suggesting that the effectiveness of the gestural features persisted even with the subtle differences between similar tongue gestures.

Differentiating normal from abnormal using the gestural descriptors. Our last experiment examined how well our SVM classifier can be trained to predict abnormal and normal tongue motion using spatio-temporal features. For this task, we collected additional data for the same set of VCV sequences that indicated whether the sequence was made with normal or abnormal speech. Using the same procedure as done in the second experiment, we performed 3 binary classification tasks, one for each of the above 3 VCV sequences. The obtained mean classification accuracies were 84,84 and $86 \%$, for /aka/, /ishi/ and /ushu/, respectively.

In summary, both velocity-based and spatio-temporal descriptors were shown to be capable of discriminating tongue motions as produced from subjects with and without speech impediments. We also found that different localized velocity-based features gave different levels of classification accuracies, suggesting that regional differences in tongue motion exist. 


\section{Conclusions}

We have presented a machine learning approach to analyze and describe motions of the human tongue as captured from dynamic US image sequences. Experimental results show that our approach of extracting and encoding the proposed descriptors from spatio-temporally aligned data is effective for automatic analysis of tongue motion as captured in US sequences. Therefore, if different levels of speech impediments lead to varying levels of changes in tongue gestures, our method may be able to differentiate between gestures of different levels of speech impediments. This would ultimately lead to the development of a set of indicators for different types of articulation disorders. We are currently pursuing the collection of such data to test this hypothesis.

\section{References}

1. Bressmann T: Ultrasound imaging and its application in speech-language pathology and speech science. Amer. Speech-Language-Hearing Assoc. Newsl. 33(4) (2007) 204-211

2. Punithakumar et al.: Detection of left ventricular motion abnormality via information measures and bayesian filtering. IEEE Trans. Inf. Technol. Biomed. 14 (2010) 1106-1113

3. Rastadmehr et al.: Increased midsagittal tongue velocity as indication of articulatory compensation in patients with lateral partial glossectomies. J Head and Neck 30(6) (2008) 718726

4. Kocjancic, T.: Ultrasound study of tongue movements in childhood apraxia of speech. In: Ultrafest V. (2010) 1-2

5. Stern, M., Hagan, J., Park, J., DanielTraub: The effects of age on tongue motion and speech duration. In: Ultrafest V. (2010) 1-2

6. Shadle, C.H., Iskarous, K., Proctor, M.I.: Use of ultrasound to study differences in the tongue dorsum of voiced vs. voiceless fricatives. In: Ultrafest V. (2010) 1-2

7. Tang, L., Hamarneh, G.: Graph-based tracking of the tongue contour in ultrasound sequences with adaptive temporal regularization. In: MMBIA. (2010) 1-8

8. Hueber et al.: Eigentongue feature extraction for an ultrasound-based silent speech interface. In: Int' Conf. on Acoustics, Speech, and Signal Proc. (2007) 1245-1248

9. Jeff Berry, Diana Archangeli, I.F.: Automatic classification of tongue gestures in ultrasound images. In: Laboratory Phonology 12. (2010)

10. Metz et al.: Nonrigid registration of dynamic medical imaging data using $\mathrm{nD}+\mathrm{t} \mathrm{B}$-splines and a groupwise optimization approach. Medical Image Analysis 15(2) (2011) 238 - 249

11. Bird, S., Leonard, J., Moisik, S.: A motion vector analysis of tongue motion in SENCOFEN /qV/ and /Vq/ sequences. In: Ultrafest V. (2010) 1-2

12. Moisik, S.R.: Laryngeal ultrasound assessment of retracted and constricted articulations by phoneticians. In: Ultrafest V. (2010) 1-2

13. Turetsky, R., Ellis, D.: Ground-truth transcriptions of real music from force-aligned midi syntheses. In: 4th ISMIR. (2003) 135-141

14. Wu, J.: A Fast Dual Method for HIK SVM Learning. In: ECCV. (2010) 552-565

15. Herold et al.: Analysis of vowel-consonant-vowel sequences in patients with partial glossectomies using 2D ultrasound imaging. In: Ultrafest V. (2010) 1-2 